\title{
Modelling the Effects of Nifedipine on Ventricular and Myometrial Cells of Pregnant Rats
}

\author{
Craig P Testrow ${ }^{1}$, Dominic G Whittaker ${ }^{1}$, Arun V Holden ${ }^{2}$, Henggui Zhang ${ }^{1}$ \\ ${ }^{1}$ The University of Manchester, Manchester, UK \\ ${ }^{2}$ University of Leeds, Leeds, UK
}

\begin{abstract}
In this study we have used computational models to investigate the effects of nifedipine on two different cell types; the rat ventricular cell and the rat myometrial cell. Nifedipine is a calcium-channel blocker commonly used by health services around the world to treat both cardiovascular conditions (such as high blood pressure) and as a tocolytic to treat pre-term birth. The latter usage is prohibited in pregnant patients with pre-existing heart conditions.

By applying discrete blocks to the L-Type calcium channels in each cell model we were able to simulate the presence of nifedipine at varying concentrations. Using the electrical and ionic responses to blocking these channels as indicators, we have been able to quantify and describe the effects of nifedipine in each cell type and compare them qualitatively.

Although any level of block will reduce the maximum level of intracellular calcium in the myometrial cell, a $60 \%$ block or higher is required to produce a change in the morphology of the calcium transient. It remains to be shown if the dose required to achieve this could result in a patient with a pre-existing heart condition experiencing hypotension or other pathological cardiac conditions during labor, if nifedipine is used as a tocolytic.
\end{abstract}

\section{Introduction}

According to the World Health Organisation, pre-term birth and the subsequent complications are the leading cause of death amongst children under the age of 5 [1]. A contemporary approach to treating pre-term birth is the application of tocolytics; drugs that inhibit uterine contractions for 24-48 hours, in order to allow time for the mother to be relocated to a facility specialised in dealing with pre-term babies. Typically the baby must be treated with steroids to aid the maturation of its lungs. New tocolytics are being developed, as none of those currently available are ideal; for instance terbutaline is known to cause cardiac arrhythmias [2], and salbutamol cannot be used on diabetic patients [3].

Nifedipine is a common tocolytic used routinely by the National Health Service. It inhibits contraction of the uterus by blocking the calcium channels of myometrial cells. However, nifedipine also has other medical applications. It is used to manage angina pectoris, high blood pressure and Raynaud's phenomenon - three cardiovascular conditions. It may even be used to treat severe high blood pressure in pregnant women. It should not be used on patients that suffer from low blood pressure, heart failure or disorders that affect muscle strength.

Pre-term birth occurs in up to $10 \%$ of pregnancies. Using nifedipine as a tocolytic on the $0.5-1.0 \%$ of pregnant patients with pre-existing cardiac conditions poses a considerable risk. For instance, treatment may result in low blood pressure which can affect blood flow to the baby.

Our investigation focuses on the behaviour exhibited by two types of cell that are affected by the application of nifedipine; ventricular myocytes and myometrial myocytes. Studying these cells simultaneously can provide insight into the effect of nifedipine on both the heart and uterus of a pregnant patient. Our objective was to investigate the characteristic behavioural changes experienced by these cells when they undergo calcium channel block.

Computational models can offer a level of control when investigating changes to ion channel behaviour that is difficult to reproduce experimentally, due to the multitude of variables that must be managed. In this study we have used two computational models; one describing a rat ventricular cell and another describing a rat myometrial cell.

\section{Methods}

The ventricle cell is described by the Pandit et al model [4] and the myometrial cell by a modified Tong et al model [5]. Figure 1 shows the two cell models used, 
including each of the ion channels, pumps and exchangers. Detailed descriptions of these models can be found in the original publications. The modifications made to the Tong et al model will be published in future articles.

The models use Hodgkin-Huxley type formulations to describe changes in the cell membrane potential (V) over time (t), known as the action potential (AP); the total membrane current $\left(\mathrm{I}_{\mathrm{tot}}\right)$ is divided by the membrane capacitance $\left(\mathrm{C}_{\mathrm{m}}\right)$ :

$$
\frac{d V}{d t}=-\frac{I_{\text {tot }}}{C_{\mathrm{m}}}
$$

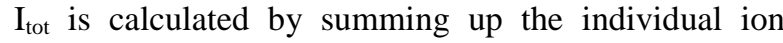
currents shown in Figure 1. The L-Type calcium current $\left(\mathrm{I}_{\mathrm{CaL}}\right)$ plays an important role in the morphology of the action potential in both cell types.
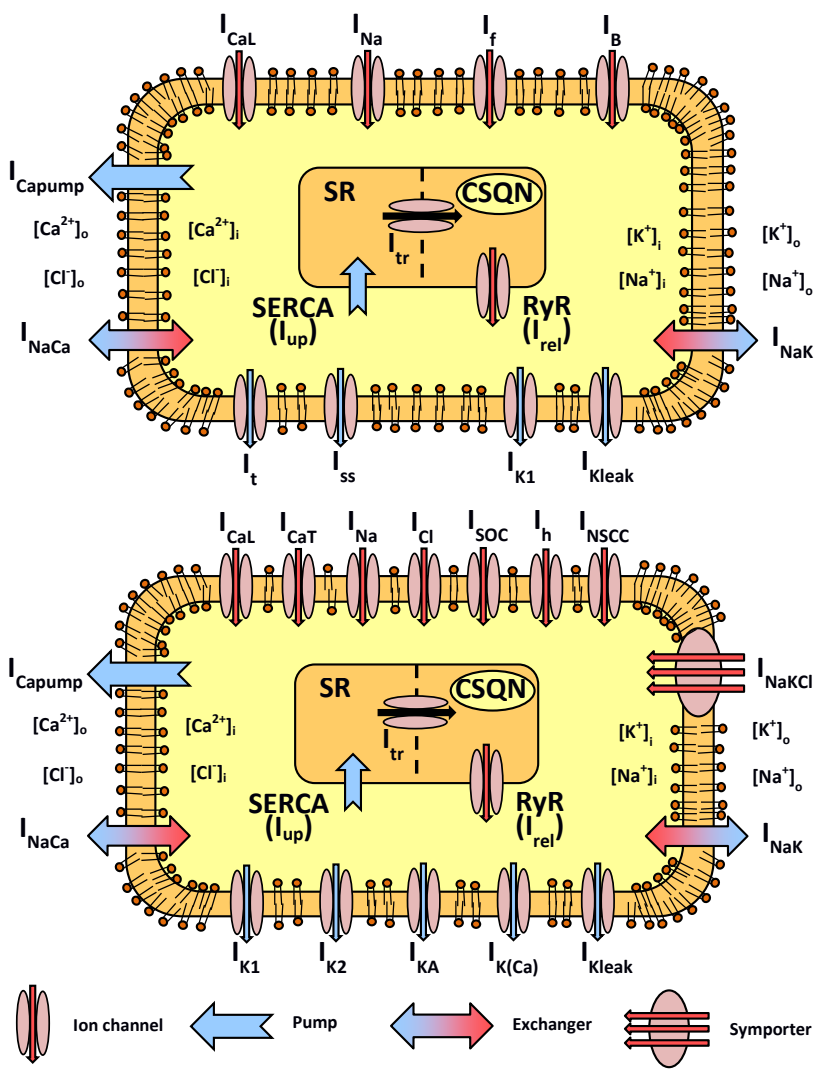

Figure 1. Schematic cell diagrams of the Pandit et al model of the ventricular cell (top) and modified Tong et al model of the myometrial cell (bottom). All the channels, exchangers and pumps are included. Red arrows indicate a flow into the intracellular space, and blue a flow out.

An AP leads to an influx of $\mathrm{Ca}^{2+}$ across the cell membrane, which in turn activates the process of calcium-induced calcium-release (CICR) from the sarcoplasmic reticulum (SR). As the concentration of calcium in the intracellular space $\left(\left[\mathrm{Ca}^{2+}\right]_{\mathrm{i}}\right)$ rises it binds to calmodulin, activating the myosin light-chain kinase (MLCK). Phosphorylated myosin can then form crossbridges with actin, causing the cell to contract. Myosin Light-Chain Phosphatase (MLCP) dephosphorylates the myosin leading to relaxation.

In each cell the effects of nifedipine were simulated by reducing the conductance of the L-Type calcium channels at regular intervals of $20 \%$. Each interval further reduces the availability of calcium in the cell, retarding the process of CICR and in turn, contraction. Observations were made of changes in the AP, $\left[\mathrm{Ca}^{2+}\right]_{i}$ and the L-Type calcium current.

The ventricle cell was paced at $1 \mathrm{~Hz}$, using a stimulus of $-2 \mathrm{pApF}^{-1}$ for $2 \mu \mathrm{s}$. The myometrial cell was stimulated at $-0.33 \mathrm{pApF}^{-1}$ for $1 \mathrm{~s}$. This would be considered a very short contraction for a laboring uterine cell. Contractions in the human heart typically last for $\sim 800 \mathrm{~ms}$, whereas uterine contractions need to build up to about 1 minute before the cervix is able to dilate. Shorter uterine contractions have been selected for this paper purely on the basis that they are easier to visualize; the behavior demonstrated by the myometrial cell in this paper is replicated in the longer contractions (Figure 4).

\section{Results}
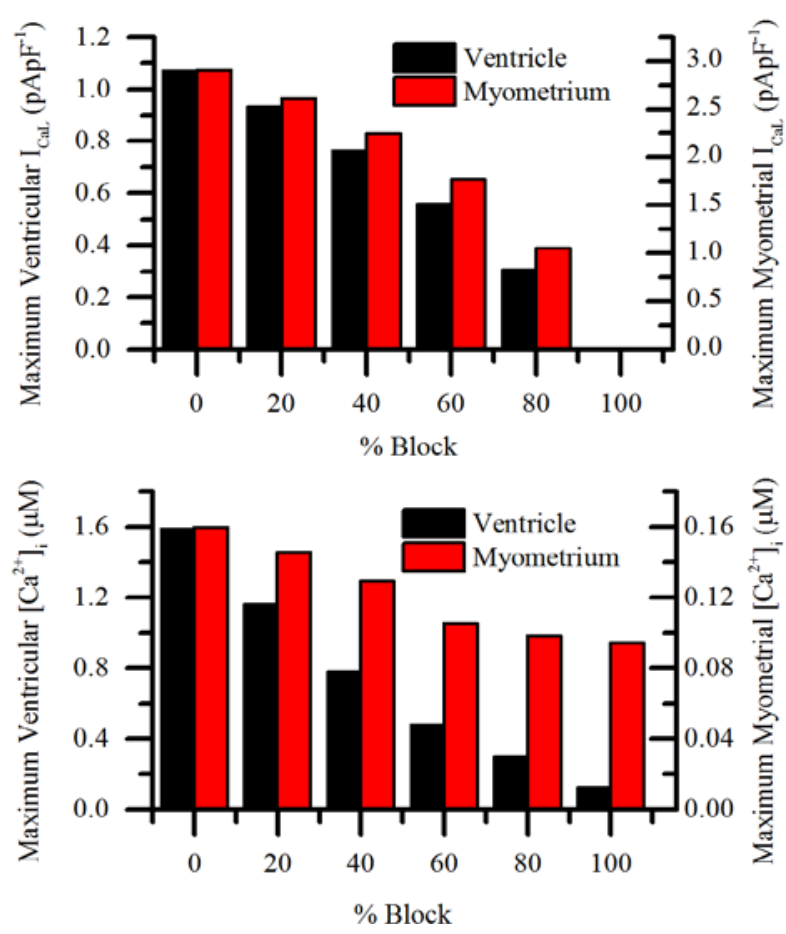

Figure 2. Changes in maximum levels of $\mathrm{I}_{\mathrm{CaL}}$ (top) and $\left[\mathrm{Ca}^{2+}\right]_{\mathrm{i}}$ (bottom) under increasing levels of nifedipine. $0 \%$ block represents the WT condition. 

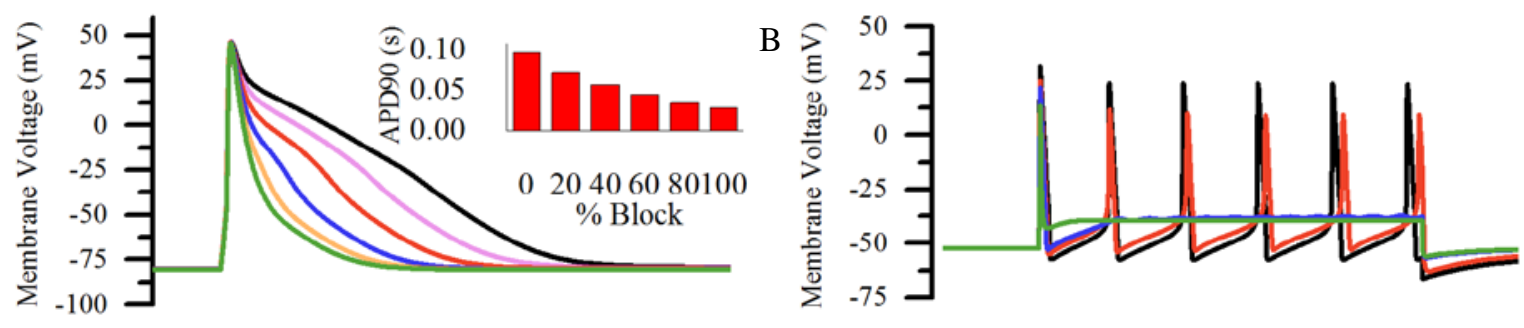

C
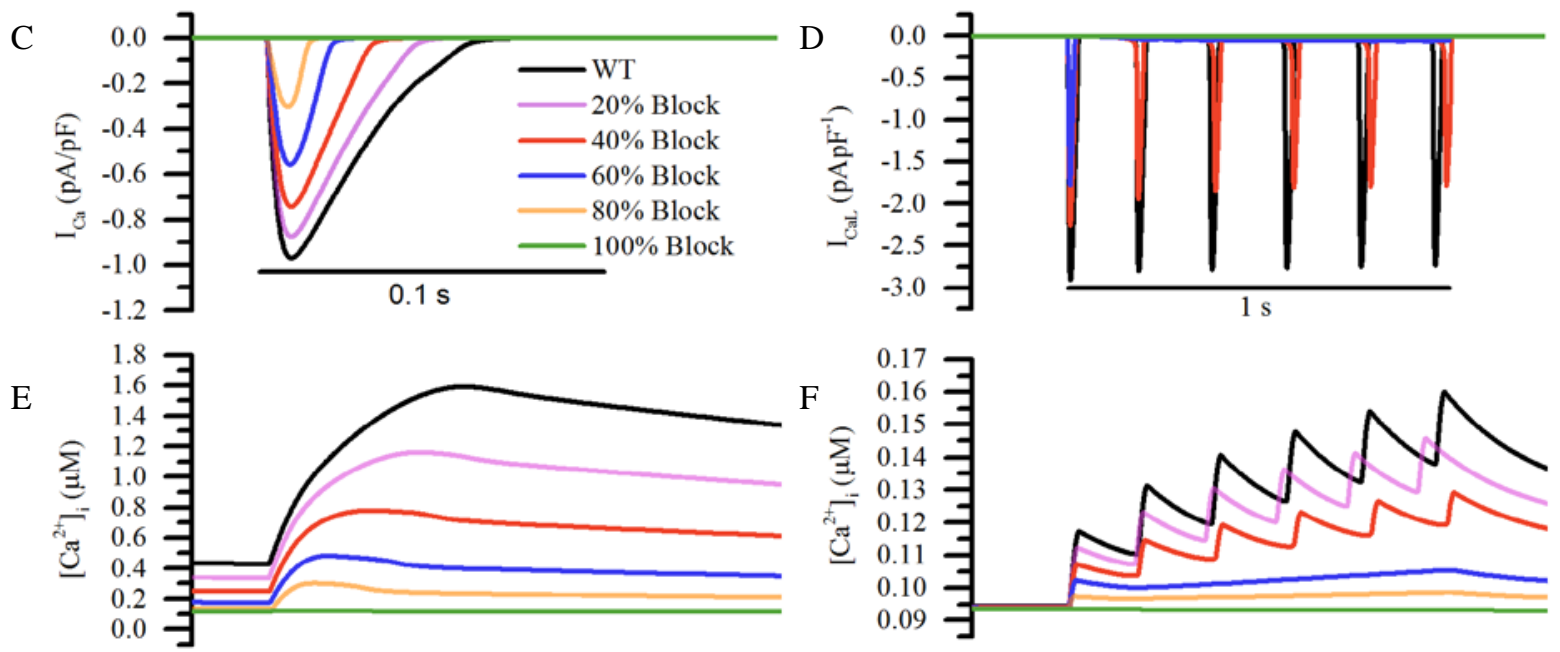

Figure 3. A comparison of the influence of nifedipine on the $\mathrm{AP}, \mathrm{I}_{\mathrm{CaL}}$ and $\left[\mathrm{Ca}^{2+}\right]_{\mathrm{i}}$ of both the ventricle (left) and myometrial (right) myocytes. A and B: the APs. C and D: $\mathrm{I}_{\mathrm{CaL}}$. E and F: $\left[\mathrm{Ca}^{2+}\right]_{\mathrm{i}} \mathrm{A}$ inset: the effect of each level of block on the APD90 of the ventricular cell.

Applying nifedipine to each model caused significant changes in their electrical and ionic behavior. According to the findings shown in Figure 2, both models trivially experience a drop in $\mathrm{I}_{\mathrm{CaL}}$ with increasing block to the LType calcium channels. However the uterine cell appears to be more resilient than the ventricular cell. This is further demonstrated by the much lower change in relative levels of $\left[\mathrm{Ca}^{2+}\right]_{i}$.

Figure 3 shows the influence of nifedipine on three important indicators of activity shared by both cell types: the AP, $\mathrm{I}_{\mathrm{CaL}}$ and $\left[\mathrm{Ca}^{2+}\right]_{\mathrm{i}}$.

The AP of the ventricular cell is formed from a quick depolarizing upstroke followed by a slower drop back to the resting potential. The rate of this drop determines the action potential duration (APD). Calcium block causes a reduction in APD (Figure 3A), as the slope of the repolarization curve is largely determined by the opposing inward $\mathrm{Ca}^{2+}$ and outward $\mathrm{K}^{+}$currents. The overall reduction in APD90 from $0 \%$ to $100 \%$ is from 94 ms to $33 \mathrm{~ms}$; a drop of $65 \%$.

$\left[\mathrm{Ca}^{2+}\right]_{\mathrm{i}}$ in the ventricular cell (Figure 3E) undergoes a steady drop in amplitude as nifedipine block increases, echoing the effect on the AP and $\mathrm{I}_{\mathrm{CaL}}$. The scale of the drop going from $0 \%$ to $20 \%$ block is $\sim 0.4 \mu \mathrm{M}$, and $\sim 0.2$ $\mu \mathrm{M}$ going from $80 \%$ to $100 \%$ block.

The myometrial AP is formed from an initial tall peak followed by a spike train with a frequency of $6 \mathrm{~Hz}$ (Figure 3B) when undergoing a constant stimulus. Up to a $40 \%$ nifedipine block it undergoes only minor changes to its frequency, with a drop of $\sim 15 \mathrm{mV}$ in amplitude. However, from a $60 \%$ block the spike train is replaced with a plateau. The amplitude of the initial peak also drops as $\mathrm{Ca}^{2+}$ block increases, by $\sim 15 \mathrm{mV}$ between $0 \%$ and $100 \%$ block.

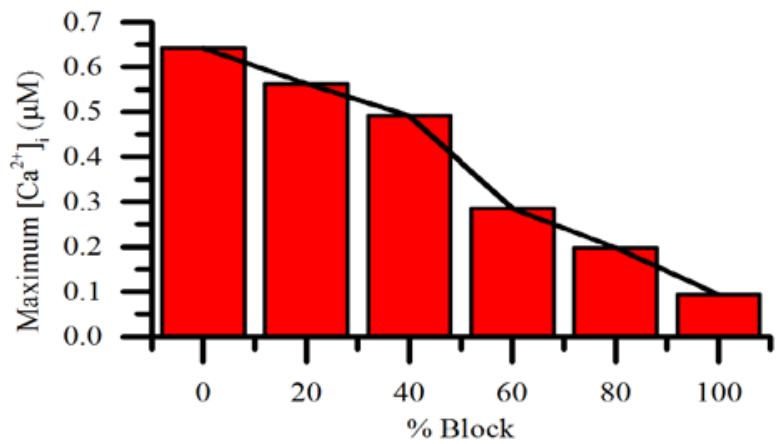

Figure 4. Maximum $\left[\mathrm{Ca}^{2+}\right]_{\mathrm{i}}$ values for myometrial cells under a 1 minute stimulus; a more realistic contraction duration. At these time scales the jump in maximum $\left[\mathrm{Ca}^{2+}\right]_{\mathrm{i}}$ between $40 \%$ and $60 \%$ block becomes more pronounced. 
The AP has a non-trivial correlation with the shape and amplitude of $\left[\mathrm{Ca}^{2+}\right]_{i}$ in the myometrial cell (Figure 3F). From $0 \%$ block to $40 \%$ block the amplitude of the AP and $\left[\mathrm{Ca}^{2+}\right]_{\mathrm{i}}$ both fall by $\sim 20 \%$. And the abolition of the plateau in the AP is mirrored in the $\left[\mathrm{Ca}^{2+}\right]_{\mathrm{i}}$. However, after $60 \%$ block the AP undergoes very minor changes, whereas the $\left[\mathrm{Ca}^{2+}\right]_{\mathrm{i}}$ continues to reduce. Once $\mathrm{I}_{\mathrm{CaL}}$ is blocked entirely $\left[\mathrm{Ca}^{2+}\right]_{\mathrm{i}}$ remains constant.

\section{Discussion}

One possible explanation for the difference in relative change of $\left[\mathrm{Ca}^{2+}\right]_{\mathrm{i}}$ in the ventricular and myometrial cells (Figure 2) is the influence of the T-Type calcium channels in the latter. The magnitude of the T-Type calcium current $\left(\mathrm{I}_{\mathrm{CaT}}\right)$ is approximately $10 \%$ of the value of $\mathrm{I}_{\mathrm{CaL}}$. This means that although it has little effect under normal conditions, it becomes increasingly influential as the $\mathrm{I}_{\mathrm{CaL}}$ is gradually blocked. $\mathrm{I}_{\mathrm{CaT}}$ is not present in the Pandit et al model as this channel has little physiological significance in the ventricles. It should be noted that levels of $\left[\mathrm{Ca}^{2+}\right]_{\mathrm{i}}$ do not increase beyond resting during the stimulated period when $\mathrm{I}_{\mathrm{CaL}}$ is blocked entirely, even with the presence of $\mathrm{I}_{\mathrm{CaT}}$ (Figure 3F).

Applying nifedipine to the ventricular cell causes reductions in APD. A shortened APD can lead to a decrease in the effective refractory period (ERP). This can have a pro-arrhythmic effect, as it increases vulnerability to sustained re-entry.

Nifedipine also causes a decrease in $\left[\mathrm{Ca}^{2+}\right]_{\mathrm{i}}$ in the ventricular cell. This leads to a decrease in force and ventricular output, potentially resulting in hypotension.

The initial peak of the myometrial AP is quite resilient to $\mathrm{Ca}^{2+}$ block; the subsequent spike train however is much more sensitive. It is useful to consider the morphology of the uterine AP in this study under two distinct domains: a spiked domain at blocks below $60 \%$ and a plateau domain above that threshold. Changes within these two domains are relatively minor.

The rapid increases in $\left[\mathrm{Ca}^{2+}\right]_{\mathrm{i}}$ caused by the spikes in the low-block domain are an important factor in the overall level of $\left[\mathrm{Ca}^{2+}\right]_{i}$. Over long action potentials a significant difference in levels of $\left[\mathrm{Ca}^{2+}\right]_{\mathrm{i}}$ will develop between the low and high-block domains (Figure 4).

If we apply this same threshold of $60 \%$ block to the ventricular myocyte we note that the effect on heart function is potentially pathological. It would experience a reduction in APD90 of $48 \mathrm{~ms}(49 \%)$ and of $1.1 \mu \mathrm{M}(69 \%)$ in $\left[\mathrm{Ca}^{2+}\right]_{\mathrm{i}}$. From this we can infer a significant drop in ventricular output may occur. However, this conclusion assumes that ventricular cells and myometrial cells are equally sensitive to nifedipine, which is almost certainly not the case. Further studies must account for any differences in potency by applying experimental findings on the IC50 values for nifedipine in each cell type.
Contemporary medical guidelines state that firstly, if nifedipine were to be used as a tocolytic on a patient with a pre-existing heart condition such as hypotension, then they are at risk of experiencing heart trouble before the dosage of nifedipine applied can reach sufficient levels to effectively halt uterine contraction.

Secondly, if nifedipine were to be used as a means of managing high blood pressure in a pregnant patient then it could potentially have the additional effect of extending labor, if taken at sufficiently high doses, which may be undesirable.

To summarize, in this study we have quantified some of the important changes that occur in both the heart and uterus of pregnant patients being treated with nifedipine. Both cell types experience reduced force output when nifedipine is applied due to the reduced intake of $\mathrm{Ca}^{2+}$ ions. There is potential for a patient to experience induced hypotension when nifedipine is used as a tocolytic to prolong labor.

\section{References}

[1] H. Blencowe, S. Cousens, M. Z. Oestergaard, D. Chou, A.B. Moller, R. Narwal, A. Adler, C. Vera Garcia, S. Rohde, L. Say, and J. E. Lawn, "National, regional, and worldwide estimates of preterm birth rates in the year 2010 with time trends since 1990 for selected countries: a systematic analysis and implications,” The Lancet, vol. 379, no. 9832, pp. 2162-2172, Jun. 2012.

[2] R. Michael Sly, J. A. Anderson, C. W. Bierman, P. Chervinsky, F. C. Cogen, J. G. Easton, I. Glazer, J. P. Kemp, A. Knight, R. A. Krumholz, R. A. Nicklas, B. M. Rudolph, D. E. Schuller, A. T. Segal, R. M. Sly, S. J. Szefler, M. J. Welch, N. J. Whitcomb, and M. Yamate, "Adverse effects and complications of treatment with betaadrenergic agonist drugs,” J. Allergy Clin. Immunol., vol. 75, no. 4, pp. 443-449, Apr. 1985.

[3] J. L. Schlienger, M. J. Compagnie, P. Dellenbach, and F. Stephan, "[Metabolic risks of salbutamol in diabetic patients. A study using somatostatin (author's transl)]," Nouv. Presse Médicale, vol. 9, no. 32, pp. 2247-2251, Sep. 1980.

[4] S. V. Pandit, R. B. Clark, W. R. Giles, and S. S. Demir, “A mathematical model of action potential heterogeneity in adult rat left ventricular myocytes,” Biophys. J., vol. 81, no. 6, pp. 3029-3051, Dec. 2001.

[5] W.-C. Tong, C. Y. Choi, S. Karche, A. V. Holden, H. Zhang, and M. J. Taggart, “A Computational Model of the Ionic Currents, Ca2+ Dynamics and Action Potentials Underlying Contraction of Isolated Uterine Smooth Muscle,” PLoS ONE, vol. 6, no. 4, p. e18685, Apr. 2011.

Address for correspondence.

Craig P. Testrow

Room 3.21, Schuster Laboratory, School of Physics and Astronomy, The University of Manchester, Oxford Road, M13 9PL

craig.testrow@manchester.ac.uk 